\title{
Evolutionary optimization of rotational population transfer
}

\author{
Arnaud Rouzée,,${ }^{1,4,}$ Omair Ghafur, ${ }^{1}$ Konstantin Vidma, ${ }^{2}$ Arjan Gijsbertsen, ${ }^{1}$ Ofer M. Shir, ${ }^{3}$ Thomas Bäck, ${ }^{3}$ Afric Meijer, ${ }^{2}$ \\ Wim J. van der Zande, ${ }^{2}$ David Parker, ${ }^{2}$ and Marc J. J. Vrakking ${ }^{1,4}$ \\ ${ }^{1}$ FOM Institute for Atomic and Molecular Physics (AMOLF), Science Park 104, NL-1098 XG Amsterdam, The Netherlands \\ ${ }^{2}$ Institute of Molecules and Materials, Radboud University Nijmegen, Toernooiveld 1, NL-6500 GL Nijmegen, The Netherlands \\ ${ }^{3}$ Leiden Institute of Advanced Computer Science (LIACS), Niels Bohrweg 1, NL-2333 CA Leiden, The Netherlands \\ ${ }^{4}$ Max Born Institut, Max Born Strasse 2A, D-12489, Berlin, Germany
}

(Received 15 October 2009; revised manuscript received 11 April 2011; published 16 September 2011)

\begin{abstract}
We present experimental and numerical studies on control of rotational population transfer of $\operatorname{NO}(J=1 / 2)$ molecules to higher rotational states. We are able to transfer $57 \%$ of the population to the $J=5 / 2$ state and $46 \%$ to $J=9 / 2$, in good agreement with quantum mechanical simulations. The optimal pulse shapes are composed of pulse sequences with delays corresponding to the beat frequencies of states on the rotational ladder. The evolutionary algorithm is limited by experimental constraints such as volume averaging and the finite laser intensity used, the latter to circumvent ionization. Without these constraints, near-perfect control ( $>98 \%)$ is possible. In addition, we show that downward control, moving molecules from high to low rotational states, is also possible.
\end{abstract}

DOI: 10.1103/PhysRevA.84.033415

PACS number(s): 33.80.-b, 33.20.Sn, 42.50.-p

\section{INTRODUCTION}

The emergence of the idea that coherent light-matter interactions allow one to exert control over the dynamics of atomic and molecular systems has changed the nature of research into these systems from passive observation to active control. One important challenge concerns the manipulation of ground-electronic-state vibrational and rotational populations since most reactions take place on the lowest energy surface. Several schemes have been proposed and tested experimentally to control the initial quantum state preparation of molecular samples. Resonant photoabsorption, two-color Raman excitation, and stimulated emission pumping are common techniques to state selectively prepare atoms or molecules prior to their use in a collision experiment. A further interesting preparation scheme is stimulated rapid adiabatic passage (STIRAP) [1], where two narrowband nanosecond lasers are used to accomplish complete population transfer from an initial state to a selected target state. STIRAP has been used to generate vibrationally pure samples [2] and can be viewed as the first example of the use of coherent control as a means to achieve quantum state preparation in a controlled manner.

Rapid progress in the development of femtosecond lasers and pulse shaping technology has helped considerably in putting ideas for controlling laser-molecule interactions into practice. Using ideas that derive from the seminal paper by Rabitz on "teaching lasers to control molecules" [3], many examples currently exist where shaped laser pulses have been optimized by a genetic or evolutionary algorithm in order to accomplish a desired task or to optimize a desired end result (see, e.g., [4-8]). The remarkable success of these experiments can be understood from the fact that feedbackcontrolled optimization drives quantum systems to perfect control, provided that no constraints are placed on the controls [9]. However, one should realize that in any experimental

\footnotetext{
*a.rouzee@amolf.nl
}

realization, a substantial number of constraints exist, such as the bandwidth of the laser, the algorithm used to control the feedback loop, and the parametrization or fidelity of the amplitude, phase, and polarization shaping applied to the laser pulse or, as we encounter in this paper, the intensity limit imposed by the ionization potential of a molecule. So far, optimal control experiments can be divided into two categories. On the one hand, there are experiments in idealized two- and three-level systems, where perfect control can be exerted that can be understood in terms of analytical solutions of the time-dependent Schrödinger equation [9]. On the other hand, there are experiments in more complex, many-level systems, where some degree of optimization is achieved, but where a mechanistic interpretation of the exerted control and an assessment of the degree of optimization are very challenging.

In this paper we present studies on rotational ladder climbing in the NO molecule (starting from a single initial rotational state) under the influence of moderately intense femtosecond laser pulses subjected to phase-shaping. We note that a rotational ladder as used here has no upper bound. Therefore, the possibility to populate only one of its rungs is certainly not obvious. Nevertheless, we show that a high degree of selectivity in the transfer of a population to a desired final state can be achieved. The degree of population transfer can be well understood if the experimental constraints are accounted for. Moreover, we show that starting from a single high rotational state, a very high degree of population transfer can be achieved toward a selected lower rotational state. This result is particularly important in the context of research on cold molecules, where a laser control scheme such as the one suggested in this study can be used to guide the molecule toward low- $J$ states.

\section{EXPERIMENTAL PROCEDURE}

The experimental setup used in these experiments has been described before [10,11]. Briefly, a state-selected molecular beam containing NO molecules in the low-field-seeking state of the $J=1 / 2 \Lambda$ doublet was generated using a hexapole 


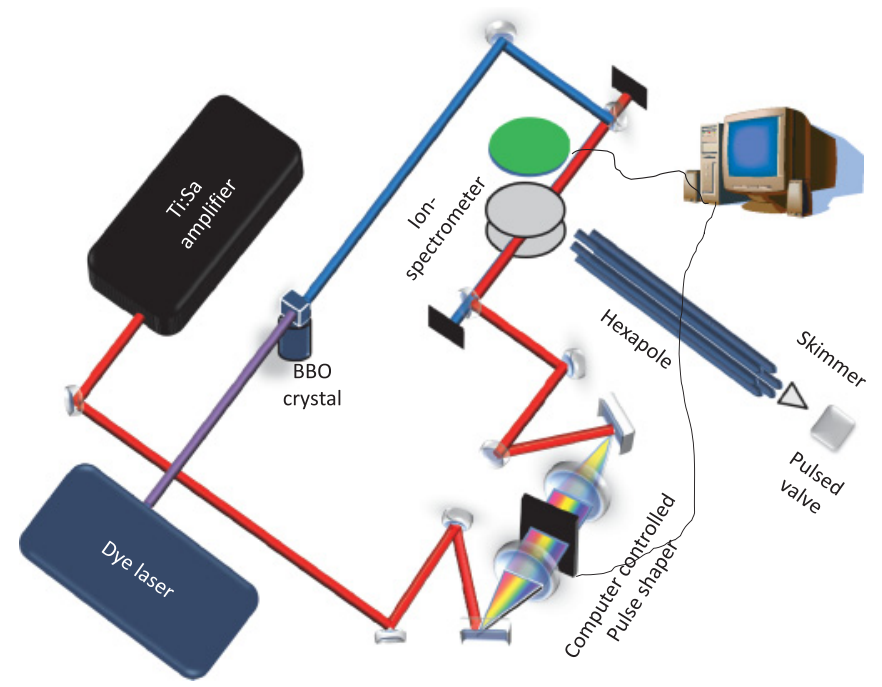

FIG. 1. (Color) Schematic of the experimental setup. It is a crossed-beam setup which combines in three perpendicular reactions the molecular beam of NO including a hexapole state selector, two counter-propagating laser beams delivering the ultrafast control pulse and a nanosecond pulse from the dye laser, and a time-of-flight direction for mass selective detection of the $m / z=30 \mathrm{NO}^{+}$ions.

state selector (see Fig. 1). The NO molecules were excited by a shaped femtosecond laser pulse that was created by passing approximately 5-mJ, 90-fs laser pulses through a computer-controlled 640-pixel spatial light modulator (SLM) placed at the center of a $4 f$ zero-dispersion line. The shaped laser pulse was focused onto the NO beam with a 400-mm lens, leading to rotational excitation via successive stimulated Raman transitions. Populations in each rotational state of NO were probed $200 \mathrm{~ns}$ after the femtosecond laser interaction using $(1+1)$ resonance-enhanced multiphoton ionization (REMPI) through the $A^{2} \Sigma^{+}$excited state of the NO molecule [12]. To this end, a 20- $\mu \mathrm{J}$, near-226-nm probe laser pulse from a frequency-doubled tunable dye laser operated at $12.5 \mathrm{~Hz}$ was used. The probe laser beam, polarized along the pump laser polarization, was focused with a 200-mm lens, ensuring a beam waist smaller than that of the pump laser. After acceleration with a $\mathrm{DC}$ field, the $\mathrm{NO}^{+}$ions resulting from ionization with the near-226-nm pulse were detected by a set of microchannel plates and then integrated using a boxcar integrator. The ion signal was then recorded at different probe wavelengths around $226 \mathrm{~nm}$ by tuning the dye laser. Optimization of the population in states $J=5 / 2$ and $J=9 / 2$ was carried out by maximizing the ion signal at a fixed wavelength of the dye laser corresponding to the spectral line $R_{21}(J=5 / 2)$ and $R_{21}(J=9 / 2)$, respectively [see Fig. 2(b)]. The algorithm used in the optimizations was the $(\mu, \lambda)$ covariance matrix adaptation evolution strategy (CMA-ES) (see, e.g., [13]). Briefly, a randomly sampled initial population of $\lambda$ candidate spectral phase functions $\phi(\omega)$ underwent experimental evaluation. The best $\mu$ phases were selected and recombined to produce new offspring. Before using these offspring, Gaussian variations, relying on auxiliary strategy parameters [14] that were self-adaptively updated, were applied. This was repeated until convergence was reached. In the reported experiments a $(\mu=8, \lambda=16)$

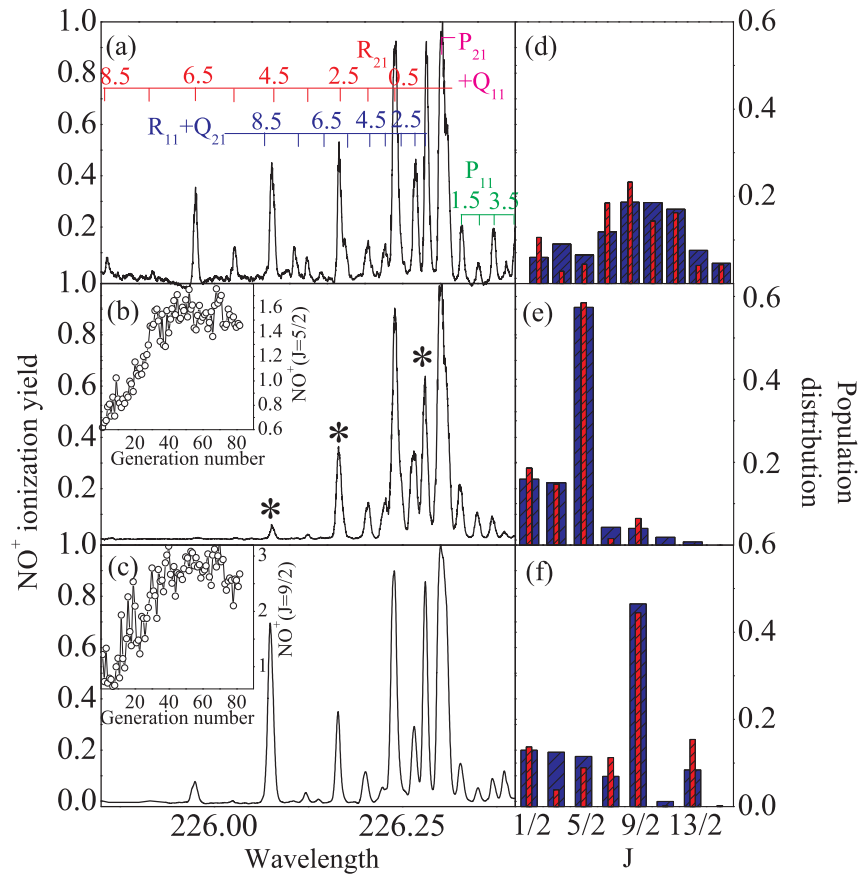

FIG. 2. (Color) (a) Measured REMPI spectrum following the excitation of hexapole state-selected $\operatorname{NO}(J=1 / 2)$ molecules by an FTL laser pulse with an estimated peak intensity of $4.2 \times 10^{13} \mathrm{~W} / \mathrm{cm}^{2}$, along with the spectroscopic assignments of the observed lines. (b) Measured REMPI spectrum at the end of an optimal control experiment targeting maximization of the product yield in $\mathrm{NO}(J=$ $5 / 2$ ); asterisks are the spectral lines used to retrieve the populations in $J=1 / 2, J=5 / 2$, and $J=9 / 2$ states. (c) Measured REMPI spectrum at the end of an optimal control experiment targeting maximization of the product yield in $\mathrm{NO}(J=9 / 2)$. Insets in (b) and (c): Evolution of the $\mathrm{NO}^{+}$ionization yield during the optimization procedure. $(\mathrm{d}-\mathrm{f}) \mathrm{NO}$ rotational population distributions derived from the measurements shown in $(\mathrm{a}-\mathrm{c})$ [wide (blue) columns], along with results obtained by solving the time-dependent Schrödinger equation for the experimental conditions used [narrow (red) columns].

strategy was deployed. To improve the convergence time, only the 250 pixels of the SLM where the majority of the pulse bandwidth was contained were varied by the algorithm.

\section{RESULTS AND DISCUSSION}

Figure 2(a) shows a recorded REMPI spectrum following the excitation of state-selected $\mathrm{NO}(J=1 / 2)$ molecules with a Fourier transform-limited (FTL) laser pulse that was focused to an intensity of $4 \times 10^{13} \mathrm{~W} / \mathrm{cm}^{2}$. When no femtosecond laser pulse is applied to the molecules (not shown here), the recorded spectrum exhibits three lines, corresponding to the $Q_{11}, Q_{21}+R_{11}$, and $R_{21}$ spectroscopic branches. As the ions are extracted in a DC electric field, the initial-state $J=1 / 2$, $\epsilon=-1$ population is partly mixed with the opposite parity state (20\%), which was taken into account in the analysis. With the laser excitation, new spectral lines appear due to the rotational population redistribution by the pump laser field. This population redistribution occurs via $\Delta J=1$ and $\Delta J=2$ transitions [11]. In order to extract the final $\mathrm{NO}(J)$ populations from this measurement, each observed spectral line was integrated over a range of $0.01 \mathrm{~cm}^{-1}$ and divided 
by the corresponding Hönl-London coefficient [15], which was corrected for laser-induced alignment [16] (note that $m$ is conserved along the pump laser polarization). The normalized distribution resulting from this procedure is shown in Fig. 2(d). The laser excitation distributes the population over a large number of rotational states, none of which accumulates more than $25 \%$ of the population. A comparison with a computed rotational population distribution is also shown [by narrow (red) columns]. A good overall agreement is found, with small discrepancies attributed to the fluctuation of the $\mathrm{NO}^{+}$signal without a dye laser.

Figure 2(b) shows the spectra obtained when optimal control was used to optimize the population ending up in $\mathrm{NO}(J=5 / 2)$, a state that can be reached by a single $\Delta J=2$ Raman transition. The optimal control procedure increases the population of this state dramatically [as shown in Fig. 2(e)]: $57 \%$ of the population ends up in the desired state after 80 generations, a threefold improvement over the best random phase function of the first generation [see inset in Fig. 2(b)] and a sixfold relative increase with respect to the population in this state using an FTL pulse with the same fluence [see Figs. 2(a) and 2(b)]. The optimization becomes more challenging when a larger number of Raman transitions are required, as in the case of $\mathrm{NO}(J=9 / 2)$. Preliminary optimizations, starting from a random initialization of $\phi(\omega)$, led to transfer of only about $35 \%$ into this state. In agreement with simulations, only a limited amount of optimization was achieved within the finite time span that the experimental conditions could be held constant ( $\leqslant 100$ generations). Suspecting that, similarly to the case of $J=5 / 2$ (see below), the optimized laser pulse for $J=9 / 2$ would consist of a sequence of pulses [17], optimizations were performed starting from oscillatory phase functions $\phi(\omega)=\alpha \sin (\beta \omega+\gamma)$ [Fig. 2(c)]. This procedure resulted in a maximum population of $46 \%$ in $\mathrm{NO}(J=9 / 2)$ [see Fig. 2(f)], which should be compared to the $10 \%$ encountered in the first generation of the aforementioned optimizations starting from random phase functions and the $20 \%$ population obtained with an FTL pulse [see Figs. 2(b) and 2(f)]. The increase of more than a factor of 2 ( $46 \%$ vs $20 \%)$ is nearly equal to the factor 3 absolute increase in signal.

In order to assess the physical mechanism underlying the observed control, cross-correlations were recorded between the optimized laser pulses and the 90-fs output of the femtosecond laser amplifier. The results of this procedure for $\mathrm{NO}(J=5 / 2)$ and $\mathrm{NO}(J=9 / 2)$ are shown in Fig. 3. In the case of $\mathrm{NO}(J=5 / 2)$, a sequence of three laser pulses is observed, separated by 2.4 and 2.8 ps with respect to the main pulse at $t=0 \mathrm{ps}$, where the first peak has a doublet structure and consists of two peaks that are separated by $400 \mathrm{fs}$. These time delay corresponds approximately to the beating period between $J=1 / 2$ and $J=5 / 2(\tau=2.6 \mathrm{ps})$ and illustrates that the evolutionary algorithm has determined a pulse shape where the Raman processes driven by the three pulses in Fig. 3(a) lead to an in-phase addition of wave function amplitude in the $\mathrm{NO}(J=5 / 2)$ state. The first pulse in the train consists of two peaks. To check whether this double-peak structure is necessary, we performed theoretically a search where we varied the timing of the two pulses with respect to the main pulse at $t=0$. Optimized population transfer was obtained only when including the double-peak structure around $-2.6 \mathrm{ps}$.

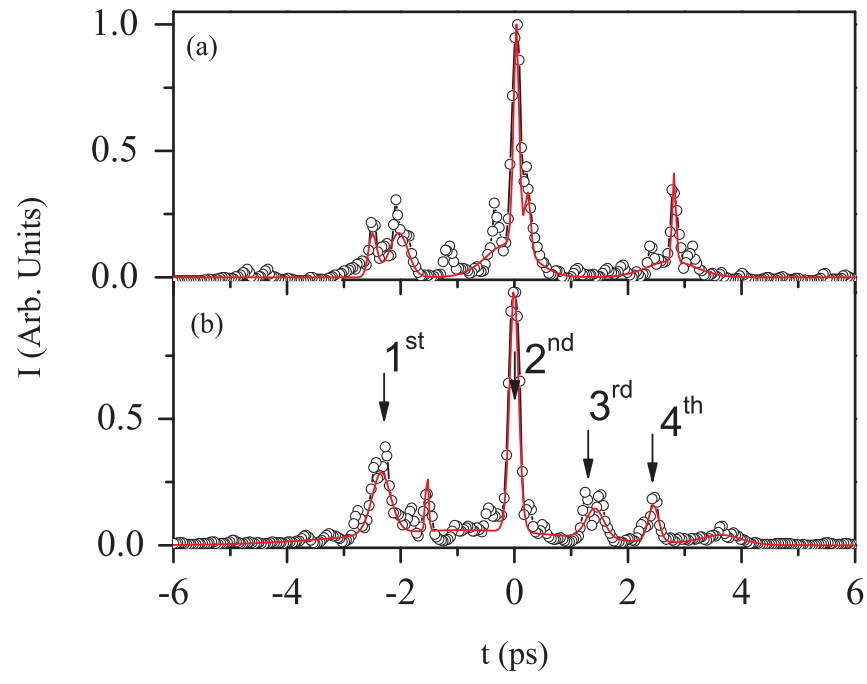

FIG. 3. (Color) (a) Optimized laser pulse resulting from an optimal control experiment targeting maximization of the product yield in $\mathrm{NO}(J=5 / 2)$; (b) the same, for maximization of the product yield in $\operatorname{NO}(J=9 / 2)$. Pulses shown in red that mimic the experimental cross-correlation were used to simulate the population distribution shown in Figs. 2(e) and 2(f).

As reported by Meijer et al. [17], a 2.6-ps timing is not optimal since it also depletes the state $J=5 / 2$ due to further excitation to the $J=9 / 2$ state. To avoid the population transfer toward higher $J$ states, the algorithm splits the first pulse in the train into two peaks, allowing maximization of the population in state $J=5 / 2$ while minimizing the population in the $J=9 / 2$ state. The optimum laser pulse shape for $\operatorname{NO}(J=9 / 2)$ is different. As before, three pulses separated by 2.4 ps can be recognized, with an additional pulse appearing about $1.3 \mathrm{ps}$ after the second, most intense pulse, that approximates the beating period between $J=5 / 2$ and $J=9 / 2$ ( $\tau=1.26 \mathrm{ps}$ ).

To test whether the observed population transfer to $\mathrm{NO}(J=$ $5 / 2)$ and $\mathrm{NO}(J=9 / 2)$ can be viewed as a satisfactory experimental outcome, optimal control calculations were performed by solving the time-dependent Schrödinger equation [11]. As in the experiment, optimizations were performed guided by the CMA-ES [13]. To reflect the pulse-shaping process implemented experimentally, the energy was kept constant during the optimization procedure and the electric field was written in the frequency domain with the phase $\phi(\omega)$ discretized into 128 parameters $\phi_{n}$ (pixels taken as a top-hat shape [18]). The maximum observed population transfer to $\mathrm{NO}(J=5 / 2)$ or $\mathrm{NO}(J=9 / 2)$ was evaluated over seven trials with the CMA-ES and plotted as a function of the peak intensity of the 90 -fs laser pulse from which the shaped laser pulses were derived. In Fig. 4 a summary of these calculations and a comparison with calculations performed using an FTL pulse are shown. The calculations were performed over an intensity range from 0 to $14 \times 10^{13} \mathrm{~W} / \mathrm{cm}^{2}$. We note that experimental optimizations could only be performed up to an intensity of $4 \times 10^{13} \mathrm{~W} / \mathrm{cm}^{2}$, since higher intensities led to unacceptable amounts of multiphoton ionization by the IR laser [19], which is not included in our numerical model. As Fig. 4 clearly shows, FTL pulses are not optimal for achieving selective population transfer, as the highest 


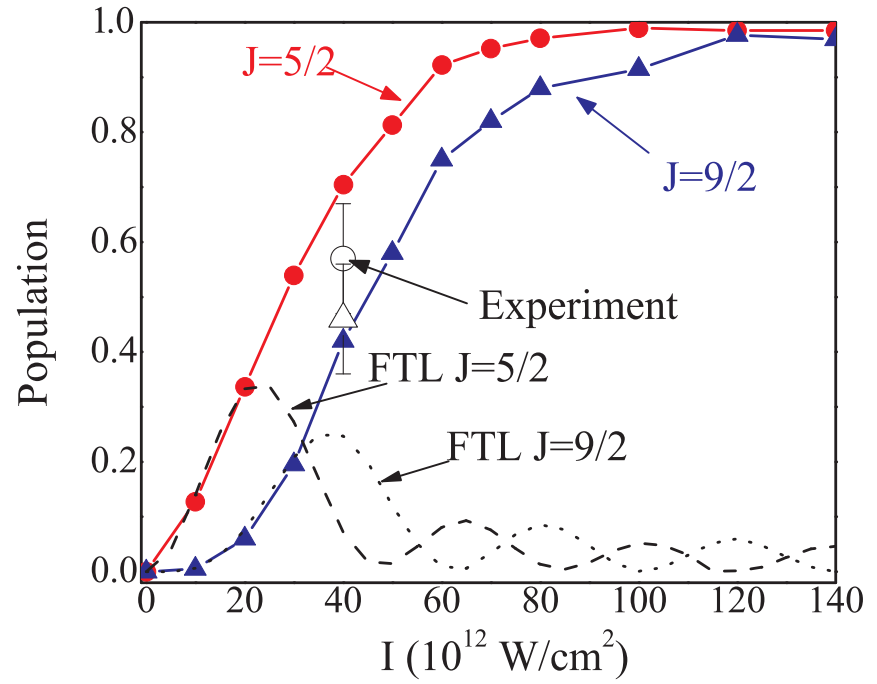

FIG. 4. (Color) Maximum product yield in $\mathrm{NO}(J=5 / 2)$ (circles) and $\mathrm{NO}(J=9 / 2)$ (triangles) encountered in seven evolutionary optimizations using the CMA algorithm, as a function of the intensity of the 90-fs laser pulse that is subjected to phase-shaping. Also shown is the population transfer to $\mathrm{NO}(J=5 / 2)$ and $\mathrm{NO}(J=9 / 2)$ for a 90-fs FTL laser pulse.

population transfer to $\mathrm{NO}(J=5 / 2)$ is only $33.8 \%$ (at a peak intensity of $\left.2.6 \times 10^{13} \mathrm{~W} / \mathrm{cm}^{2}\right)$, while the highest population transfer to $\mathrm{NO}(J=9 / 2)$ is only $25 \%$ (at an intensity of $4 \times 10^{13} \mathrm{~W} / \mathrm{cm}^{2}$ ). When increasing the intensity of an FTL pulse, the population distribution is spread over a large range of rotational states resulting in a drop in transfer efficiency to a particular state. However, when increasing the intensity, the CMA-ES procedure consistently leads to a better population transfer to a target state. The highest population that we observed was $98.9 \%$ for optimizations targeting $\mathrm{NO}(J=5 / 2)$ and $97.7 \%$ for optimizations targeting $\mathrm{NO}(J=9 / 2)$. Figure 4 suggests that without the constraint imposed by the finite pulse energy, a $100 \%$ yield may be possible [14], a remarkable result in an infinite ladder system. At an intensity of $4 \times 10^{13} \mathrm{~W} / \mathrm{cm}^{2}$, which is our best estimate of the intensity reached in the experiment when the laser pulse is compressed to a FTL laser pulse, the highest yield in our optimal control simulations targeting $\mathrm{NO}(J=5 / 2)$ and $\mathrm{NO}(J=9 / 2)$ is $70.4 \%$ and $42 \%$, respectively. Considering the uncertainty in the intensity as well as the intensity fluctuations, these values are quite close to those obtained experimentally. We note that the pulse shapes found experimentally and theoretically (see Fig. 5) are very similar. In the case of $J=5 / 2$, the pulse shape is dominated by three peaks separated by $2.6 \mathrm{ps}$, with a similar substructure around the first pulse at $-2.6 \mathrm{ps}$. This solution is, moreover, quite robust since the algorithm always found this pulse sequence over the seven runs performed. Upon increasing the intensity in our calculations, this robustness vanishes. The solutions found by the algorithm become rather complicated and are different for each run. Each run still achieves a very high degree of population transfer. Our experimental results in which a unique solution is found show that constraints not only impact the efficiency of the optimization process, but also impose limitations on the controllability of the system,

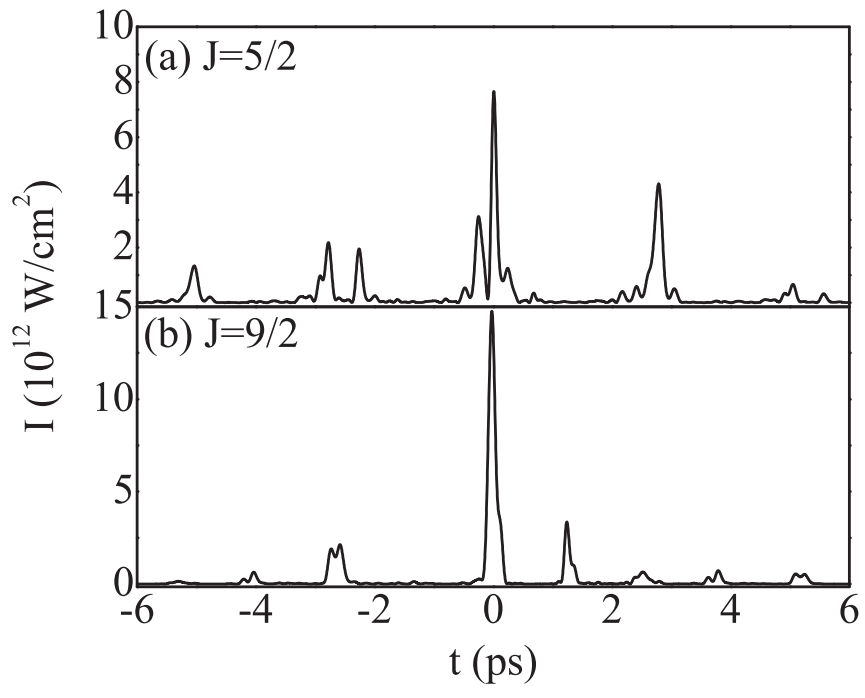

FIG. 5. Optimized laser pulse resulting from an optimal control simulation targeting maximization of the product yield in $\mathrm{NO}(J=$ $5 / 2)$ (a) and in $\operatorname{NO}(J=9 / 2)$ (b). Optimization is performed under the constraint of a constant energy, with a maximum energy that corresponds to an FTL pulse of $4 \times 10^{13} \mathrm{~W} / \mathrm{cm}^{2}$.

influencing the number of qualitatively different solutions that can accomplish the task at hand [9].

One may ask if a high controllability is also achievable when starting from a high rotational state and attempting a downward transfer. Transferring the rotational population from a high- $J$ state to a lower rotational state is interesting in the context of the production of cold molecular samples. The production of translationally cold NO molecules was shown by Chandler and co-workers [20]. In that experiment, a translationally cold sample of NO molecules results from inelastic collisions with argon atoms. Essentially all slow NO molecules in the experiment by Chandler et al. are in the $J=15 / 2, m_{a}=1 / 2$ state, where $m_{a}$ is the projection of $J$ on the kinematic apse $\hat{a}$, defined as the direction of the difference between the momentum after and that before collision. With a
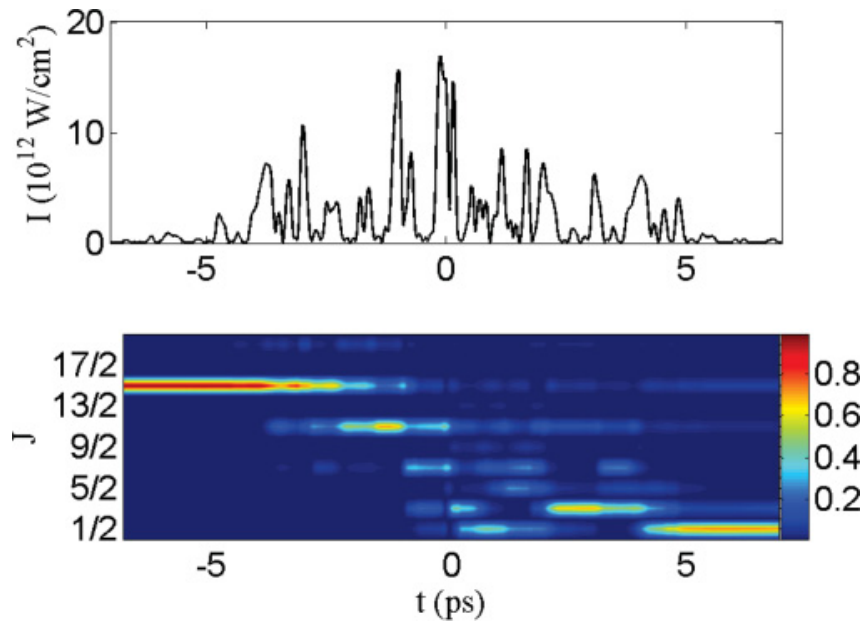

FIG. 6. (Color) Top: Optimized laser pulse resulting from an optimal control simulation targeting maximization of the product yield in $\mathrm{NO}(J=1 / 2)$ starting from $J=15 / 2$. Bottom: Corresponding time-dependent population distribution during the field. 
linearly polarized laser pulse, $m$ is conserved; in general, this complicates cooling using shaped intense laser pulses, but by choosing the laser polarization along the kinematic apse, the desired transition is $(J=15 / 2, m=1 / 2) \rightarrow(J=1 / 2, m=$ $1 / 2$ ). This is just the opposite of the rotational ladder climbing discussed in the first part of this work. Also, in this case, ionization imposes a restriction on the maximum laser intensity in the optimized pulse, which complicates downward transfer from $\mathrm{NO}(J=15 / 2)$. To demonstrate downward transfer using a shaped laser pulse, Fig. 6 shows the result of a simulation aiming to optimize the population of NO molecules in the $J=1 / 2$ state starting from molecules initially prepared in the $J=15 / 2, m=1 / 2$ state. In this simulation, we have used a penalty factor proportional to the ionization rate of NO [19]. As Fig. 6 shows, $73.4 \%$ of the population was transferred to $J=1 / 2$ for a resulting electric field with a peak intensity of $1.7 \times 10^{13} \mathrm{~W} / \mathrm{cm}^{2}$. A similar simulation starting from $J=9 / 2$ yielded the transfer of $97 \%$ of the population into $J=1 / 2$. If no ionization restriction is applied, essentially all molecules can be transferred down to the desired state. However, as shown in Fig. 6, the pulse shape found by the algorithm is very rich. This result illustrates the complexity of an open rotational quantum ladder system, where, even in the case of a diatomic molecule, a large number of states are coupled.

In conclusion, we have shown that a high degree of rotational-state selectivity can be achieved in many-level open systems using a shaped laser pulse. The degree of selectivity is limited in our experiment by the maximum intensity that can be used. Controlling the rotational-state distribution starting from a high rotational state is also feasible. This may be important in the context of cold molecule research, where the ability to actively guide the population from high rotational states to the ground rotational state of the molecule can help in the preparation of ultracold molecular samples.

\section{ACKNOWLEDGMENTS}

This work is part of the research program of the Stichting voor Fundamenteel Onderzoek der Materie (FOM), which is financially supported by the Nederlandse organisatie voor Wetenschappelijk Onderzoek (NWO).
[1] U. Gaubatz, P. Rudecki, S. Schiemann, and K. Bergmann, J. Chem. Phys. 92, 5363 (1990).

[2] S. Schiemann, A. Kuhn, S. Steuerwald, and K. Bergmann, Phys. Rev. Lett. 71, 3637 (1993).

[3] R. S. Judson and H. Rabitz, Phys. Rev. Lett. 68, 1500 (1992).

[4] A. Assion, T. Baumert, M. Bergt, T. Brixner, B. Kiefer, V. Seyfried, M. Strehle, and G. Gerber, Science 282, 919 (1998).

[5] R. J. Levis, G. M. Menkir, and H. Rabitz, Science 292, 709 (2001).

[6] T. Brixner, N. H. Damrauer, P. Niklaus, and G. Gerber, Nature 414, 57 (2001).

[7] R. Bartels, S. Backus, E. Zeek, L. Misoguti, G. Vdovin, I. P. Christov, M. M. Murnane, and H. C. Kapteyn, Nature 406, 164 (2000).

[8] J. L. Herek, W. Wohlleben, R. J. Cogdell, D. Zeidler, and M. Motzkus, Nature 417, 533 (2002).

[9] H. A. Rabitz, M. M. Hsieh, and C. M. Rosenthal, Science 303, 1998 (2004).

[10] A. Gijsbertsen, W. Siu, M. F. Kling, P. Johnsson, P. Jansen, S. Stolte, and M. J. J. Vrakking, Phys. Rev. Lett. 99, 213003 (2007).
[11] O. Ghafur, A. Rouzée, A. Gijsbertsen, W. Siu, S. Stolte, and M. J. J. Vrakking, Nat. Phys. 5, 289 (2009).

[12] H. Hasegawa and Y. Ohshima, Phys. Rev. A 74, 061401 (2006).

[13] N. Hansen and A. Ostermeier, Evolut. Comput. 9, 159 (2001).

[14] O. M. Shir, V. Beltrani, T. Bäck, H. Rabitz, and M. J. J. Vrakking, J. Phys. B 41, 074021 (2008).

[15] R. N. Zare, Angular Momentum: Understanding Spatial Aspects in Chemisty and Physics (John Wiley \& Sons, New York, 1988).

[16] D. C. Jacobs and R. N. Zare, J. Chem. Phys. 85, 5457 (1986)

[17] A. S. Meijer, Y. Zhang, D. H. Parker, W. J. van der Zande, A. Gijsbertsen, and M. J. J. Vrakking, Phys. Rev. A 76, 023411 (2007).

[18] E. Hertz, A. Rouzée, S. Guérin, B. Lavorel, and O. Faucher, Phys. Rev. A 75, 031403 (2007).

[19] A. Talebpour, S. Larochelle, and S. L. Chin, J. Phys. B 30, L245 (1997).

[20] M. S. Elioff, J. J. Valentini, and D. W. Chandler, Science 302, 1940 (2003). 